\title{
High-resolution three-dimensional reconstruction of a whole yeast cell using focused-ion beam scanning electron microscopy
}

Dongguang Wei ${ }^{1}$, Scott Jacobs ${ }^{2}$, Shannon Modla ${ }^{2}$, Shuang Zhang ${ }^{3}$, Carissa L. Young ${ }^{4}$, Robert Cirino ${ }^{2}$, Jeffrey Caplan $^{2}$, and Kirk Czymmek ${ }^{2,5}$

${ }^{1}$ Carl Zeiss Microscopy, LLC. One Zeiss Drive Thornwood, NY, USA, ${ }^{2}$ UD Bio-Imaging Center, Delaware Biotechnology Institute, University of Delaware, 15 Innovation Way, Newark, DE, USA, ${ }^{3}$ Visualization Sciences Group, 15 New England Executive Park, Burlington, MA, USA, ${ }^{4}$ Department of Chemical and Biomolecular Engineering, University of Delaware, 150 Academy Street, Newark, DE, USA, and ${ }^{5}$ Department of Biological Sciences, University of Delaware, 330 Wolf Hall, Newark, DE, USA

BioTechniques 53:41-48 (July 2012) doi 10.2144/000113850

Keywords: Focused-ion beam scanning electron microscopy; whole cell; 3D reconstruction; yeast; organelle connectivity

Supplementary material for this article is available at www.BioTechniques.com/article/113850

We developed an approach for focused gallium-ion beam scanning electron microscopy with energy filtered detection of backscattered electrons to create near isometric voxels for high-resolution whole cell visualization. Specifically, this method allowed us to create three-dimensional volumes of high-pressure frozen, freeze-substituted Saccharomyces cerevisiae yeast cells with pixel resolutions down to $3 \mathrm{~nm} /$ pixel in $\mathrm{x}$, y, and $\mathrm{z}$, supported by both empirical data and Monte Carlo simulations. As a result, we were able to segment and quantify data sets of numerous targeted subcellular structures/organelles at high-resolution, including the volume, volume percentage, and surface area of the endoplasmic reticulum, cell wall, vacuoles, and mitochondria from an entire cell. Sites of mitochondrial and endoplasmic reticulum interconnectivity were readily identified in rendered data sets. The ability to visualize, segment, and quantify entire eukaryotic cells at high-resolution (potentially sub-5 nanometers isotropic voxels) will provide new perspectives and insights of the inner workings of cells.

Elucidating the three-dimensional (3D) spatial distribution of structures and molecules within cells and tissues is critical for determining the nature of numerous cellular processes. Consequently, a multitude of technologies using photons and electrons (1-3) as well as $\mathrm{x}$-rays (4) have been developed to permit the 3D spatial visualization of organisms, tissues, cells and sub-cellular constituents. In particular, focused ion beam scanning electron microscopy (FIB-SEM) has drawn significant attention as a powerful technology for creating 3D volumes of biological specimens at electron microscopy resolution $(5,6)$. To date, the majority of FIB-SEM approaches have employed variations in conventional chemical fixation that include an aldehyde primary fixation in combination with osmium tetroxide and uranyl acetate en bloc staining $(6,7)$ while others $(8,9)$ also included thiocar- bohydrazide-osmium ligand treatment (10), potassium ferrocyanide $(9,11-14)$, and/or tannic acid $(5,14)$ for enhanced contrast. In one instance, pre-fixation in paraformaldehyde prior to cryo-preservation with high-pressure freezing was employed, but no differences were observed compared with conventional fixation, thus cryo-data data was not shown (15). FIB-SEM of biological samples has been demonstrated at $~ 50-60 \mathrm{~nm}(5,9,13-14), 30-40 \mathrm{~nm}$ (11) and down to $\sim 18-20 \mathrm{~nm}$ slice z-intervals in mammalian cells $(6,15,16)$ and $100 \mathrm{~nm}$ z-interval specifically in yeast (7) which are comparable to serial sectioning methods or block-face imaging using diamond knives $(1,17)$. More recently, the limits of FIB-SEM has been even further pushed with FIB conditions set to acquire $5 \mathrm{~nm}$ isotropic voxels in brain tissue (12). For high-resolution 3D imaging of biological samples, transmission electron microscopy
(TEM) tomography is the method of choice for acquiring cellular structures down to $\sim 3 \mathrm{~nm}$ resolution (18), including yeast (19); FIB milling in conjunction with TEM tomography has been shown as a powerful enhancement to cryo-TEM tomography (8). However, TEM tomography is typically limited to 0.5 micron thick sections, making reconstruction of an entire eukaryotic cell technically very challenging. In an attempt to approach the resolution of TEM tomography using FIB-SEM for whole cell reconstructions, we pushed the limits of focused ion beam milling and backscattered electron (BSE) detection to obtain ca. $3 \mathrm{~nm}$ isotropic voxels. This significant z-resolution improvement enabled us to create high-resolution $5 \mathrm{~nm}$ x $5 \mathrm{~nm} \times 5 \mathrm{~nm}$ isotropic renderings and quantification of an entire cell, including numerous subcellular structures, with the yeast model organism, Saccharomyces 
cerevisiae. The purpose of this work was to optimize cryo-fixation sample preparation, milling and imaging conditions to quantify and document the 3D spatial distribution of sub-cellular structures from an entire single cell at nanoscale resolutions.

\section{Materials and methods}

Cell growth

S.cerevisiae yeast strains BJ5464 and BY4742 grown in YPD were used in this study. Overnight cultures were grown to mid-log phase $(0.8 \leq \mathrm{OD} \leq 2.0)$ in $500 \mathrm{mLs}-1$ L of YPD media $\left(30^{\circ} \mathrm{C}, 275 \mathrm{rpm}\right)(20-21)$. YPD medium was prepared as described (22). To contrast the effects of cellular stress induced by chemical treatment (23), $8 \mathrm{mM}$ of dithiothreitol (DTT) (Sigma-Aldrich, St. Louis, MO, USA) was added to BY4742 ( $5 \mathrm{~nm}$ isotropic data set only). Following incubation for $2 \mathrm{~h}$ at $30^{\circ} \mathrm{C}$ and $275 \mathrm{rpm}$, unstressed and stressed BY4742 cells were harvested. Cells were centrifuged at 2,500 rcf for $5 \mathrm{~min}$ at $4^{\circ} \mathrm{C}$ for subsequent cryopreservation.

Electron microscopy sample preparation A yeast paste was high pressure frozen in a Leica EMPact in $1.2 \mathrm{~mm} \times 200 \mu \mathrm{m}$ hats and freeze substituted at $-80^{\circ} \mathrm{C}$ in $2 \%$ osmium tetroxide in acetone containing $1 \%$ water for 3-4 days as described previously (24). It must be noted, that even with freeze-substitution, some yeast membranes are difficult to contrast (25). Samples were warmed to $-20^{\circ} \mathrm{C}$ over a $16 \mathrm{~h}$ period, to $4^{\circ} \mathrm{C}$ for $3 \mathrm{~h}$, to room temperature for $2 \mathrm{~h}$ and washed in acetone. Yeast were en bloc stained in saturated uranyl acetate in 100\% acetone overnight, washed in acetone, transferred to 1:1 ethanol:acetone and en bloc stained with saturated lead acetate in 1:1 ethanol: acetone for 2-4 h (26). Samples were dehydrated in acetone and infiltrated with Embed-812 epoxy resin, embedded in BEEM capsules and polymerized for $48 \mathrm{~h}$ at $60^{\circ} \mathrm{C}$. All samples were evaluated on a Zeiss LIBRA 120 Plus transmission electron microscope with a Gatan Ultrascan $10002 \mathrm{k}$ x $2 \mathrm{k}$ camera prior to FIB analysis.

\section{FIB/SEM image stack acquisition}

Cured resin blocks were manually trimmed to expose yeast cells and a glass knife was used to face the block. The final block size was trimmed as small as possible (about $1 \mathrm{~mm}$ in size) so that sample drift from continuous degassing of resin was minimized. The block was then attached to an aluminum SEM stub with silver paint. The block and stub was coated with Pt for 5 min resulting in a continuous conductive $\mathrm{Pt}$ layer about $100 \mathrm{~nm}$ thick. This continuous Pt layer served as a medium for dissipating charge and heat generated from the electron and gallium ion beams interacting with the sample block. A Zeiss Auriga FIB/SEM $\mathrm{XBeam}$ system that is equipped with a Cobra-focused gallium ion beam column, a Schottky field emission gun and a Gemini electron column was employed for all the milling and imaging work in this study. For imaging and milling, the sample was tilted to a $54^{\circ}$ angle and placed at a working distance about $5 \mathrm{~mm}$ below the SEM final lens so that the sample surface was perpendicular to the ion beam where the electron beam and ion beam meet. The electron beam (SEM imaging) was used to locate a site of interest and then the ion beam (FIB) was used to prepare the block face for automated image stack acquisition. For FIB milling, we employed a $30 \mathrm{keV}$ Ga ion beam with a $12 \mathrm{nA}$ current to cut a trench so that the $3 \mathrm{D}$ imaging targeted volume was exposed and the surface was prepared to be milled and imaged. This also helped accommodate some re-deposited materials and reduced the possibility that neighboring materials would interfere with the passing of BSEs to the detector. To reduce the damage caused by large current FIB milling, the periphery of the volume of interest was cleaned with a $1 \mathrm{nA}$ beam. A $600 \mathrm{pA}$ beam was used for final 3D data acquisition.

Image stack acquisition was executed in an automated fashion where both ion milling parameters (i.e., probe current, dwell time and milling interval (z-interval)) and SEM imaging parameters (i.e., beam energy and current, dwell time, pixel size, detector selection and energy filtering grid bias, focus and stigmator settings) were set before the process started. Specifically, all images were taken with a beam energy at 1.5 $\mathrm{keV}$, and probe current of $931 \mathrm{pA}(60 \mu \mathrm{m}$ aperture and high current mode), working distance at $5.0 \mathrm{~mm}$ and tilt angle at $54^{\circ}$. Line averaging was used for noise reduction $(\mathrm{N}=30)$ at an image store resolution of $3073 \times 2304$ with a dwell time of 12.7 $\mu \mathrm{sec} / \mathrm{pixel}$, resulting in a total cycle time of around $1.5 \mathrm{~min} /$ image. Electron dosage per square nanometer was calculated at total electrons per square nanometer $(30$ line passes in "line average"), or electrons per square nanometer per pass. For the 3 $\mathrm{nm}$ data set, it was 8220 electrons $/ \mathrm{nm}^{2}$ and 274 electrons $/ \mathrm{nm}^{2} /$ pass, the $5 \mathrm{~nm}$ data set 2958 electrons $/ \mathrm{nm}^{2}$ and 99 electrons $/ \mathrm{nm}^{2} /$ pass and the $15 \mathrm{~nm}$ data set 329 electrons/ $\mathrm{nm}^{2}$ and 11 electrons $/ \mathrm{nm}^{2} /$ pass. The energy filtering grid was set at $500 \mathrm{eV}, 750 \mathrm{eV}$ and $1000 \mathrm{eV}$ for the data sets with $15 \mathrm{~nm}, 5 \mathrm{~nm}$, and $3 \mathrm{~nm}$ z-interval, respectively. A typical stack of images with an $x-y-z$ pixel size of 5 nm covered a volume of $\sim 8 \mu \mathrm{m} \times 10 \mu \mathrm{m} \times$ $8 \mu \mathrm{m}$ and took about $35 \mathrm{~h}$.

Normal FIB artifacts such as curtaining were minimized by careful preparation of the block face and deposition of surface protection layer (Pt or C). Surface charging was also reduced by recoating the sample after a trench was FIB-cut (making the side walls of the cut block conductive) and by using the energy filter to block the lower energy BSEs (more susceptible to surface charges) from reaching the Energy Selective Backscattered electron (EsB) detector. We also noted that blanking the electron beam during FIB milling allowed us to obtain higher quality images. Through long acquisitions hours, image drift was an unavoidable phenomenon and was minimized by the following steps: (i) we cut the embedded sample block as small as possible $(\sim 1 \mathrm{~mm}$ cube) and pre-evacuated the sample for at least $24 \mathrm{~h}$ before starting FIB sectioning and imaging, (ii) we mounted the sample on a stub with silver paint around it, (iii) we coated the sample with about $10-15 \mathrm{~nm}$ thick Pt layer using a Cressington Sputter Coater to make the surface as conductive as possible in order to avoid surface charge induced image drift, and (iv) we allowed at least one hour for the sample to achieve equilibrium (mechanically and thermally) after the sample was inserted and the site of interest was found.

\section{Stack alignment, segmentation, and $3 \mathrm{D}$ presentation}

Once an image stack was acquired, alignment of the image stack (See Supplementary Material for additional details), noise reduction, reconstruction of a voxel image from the aligned image stack, segmentation based on intensity and/or shape of known organelle structure and the final presentation in $3 \mathrm{D}$ space was generated. For the cell FIB sectioned at a $10 \mathrm{~nm}$ isotropic pixel resolution, the ER was segmented with Avizo software application framework whereas all other organelles were segmented in $\operatorname{IMOD}(5,27)$. All other data sets were processed exclusively using Avizo with a customized workflow supporting individual cell segmentation. Segmentations were based on intensity and known morphology of the target structure. Segmentation results were used to reconstruct the geometrical representations of the organelles and to extract quantitative information. Avizo was also used to generate all pictures and movies.

\section{Monte Carlo simulations}

When a primary electron beam penetrates into solid sample surface, backscattering occurs. The BSEs carry energy below the 

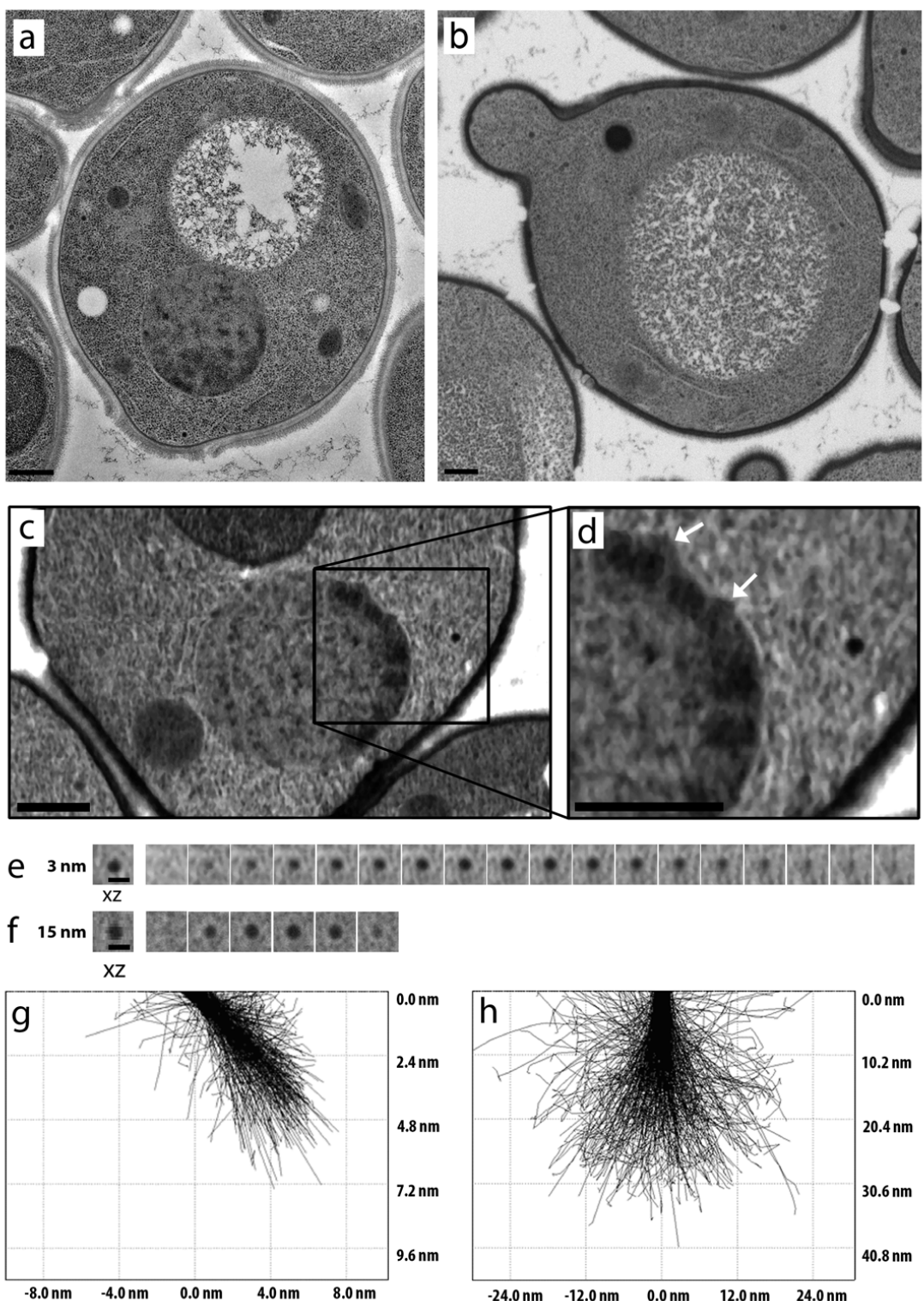

Figure 1. Enhanced FIB resolution. (A) Transmission electron micrograph of Saccharomyces cerevisiae. Scale bar equals $500 \mathrm{~nm}$. (B) Comparable SEM micrograph. Scale bar equals $500 \mathrm{~nm}$. (C) XZ crosssection of a $\sim 3 \mathrm{~nm}$ isotropic FIB data set. Scale bar equals $500 \mathrm{~nm}$. (D) Magnified view of boxed area (black) from panel (C). Arrows indicated nuclear pores. Scale $=500 \mathrm{~nm}$. (E) Left panel shows an XZ view of a $61 \mathrm{~nm}$ vesicle at $3 \mathrm{~nm}$ z-interval. Right panels show 18 serial $X Y$ images at $3 \mathrm{~nm}$ z-interval through the vesicle. Scales $=100 \mathrm{~nm}$. (F) Left panel shows XZ view of a $74 \mathrm{~nm}$ vesicle at $15 \mathrm{~nm}$ z-interval. Right panels show 6 serial XY images at $15 \mathrm{~nm}$ z-interval through the vesicle. (G) Monte Carlo simulation of electron trajectory within epoxy with $1 \%$ (atom)Os at median energy (250 eV) of the energy filtering window: $500 \mathrm{eV}$, primary beam energy $\left(\mathrm{E}_{0}\right): 1500 \mathrm{eV}$, filtering grid bias $\left(\mathrm{E}_{\text {grid }}\right): 1000 \mathrm{eV}$, sample tilt (T): $54^{\circ}, 500$ electrons. (H) Electron trajectory without tilt or energy filter - parameters used same as in $(G)$, but calculated at median energy at $750 \mathrm{eV}$ and $\mathrm{T}=0^{\circ}$.

primary beam energy due to inelastic interactions between the incident electrons and the solid sample. The distance that the BSEs travel within the sample back toward the surface follows nearly a linear relationship with the energy they carry $(28,29)$ and can be calculated with Monte Carlo simulation using CASINO v2.4.8.1. In this work, the primary beam energy was set at 1500 $\mathrm{eV}$ and a bias energy was applied to the energy filtering grid thus preventing BSEs of energy below the set bias (for example, $1000 \mathrm{eV}$ ) to reach the detector (Supplementary Figure 2). Therefore, only BSEs carrying energy above the set bias energy reached the detector. We conservatively calculated the stopping range of electrons in the simulated yeast sample [Epoxy resin $\left(\mathrm{C}_{21} \mathrm{H}_{25} \mathrm{ClO}_{5}\right)$ with $1 \%$ (atom) Os)] at energy filtering biases of $1000 \mathrm{eV}$ and 0 $\mathrm{eV}$ (no filter, conventional BSE detection system) respectively, using median values of the selected energy windows, for instance, $250 \mathrm{eV}$ for the set bias of $1000 \mathrm{eV}$ (e.g., energy window width of $500 \mathrm{eV}$ ) and 750 $\mathrm{eV}$ for no filter (e.g., primary beam energy, $1500 \mathrm{eV}$ ). Figure $1 \mathrm{~g}$ showed the electron trajectory at $54^{\circ}$ tilt with $1000 \mathrm{eV}$ bias and Figure $1 \mathrm{~h}$ showed the electron trajectory when no filtering was applied at $0^{\circ}$ tilt.

\section{Results and discussion}

To achieve optimal morphology and contrast by EM, S. cerevisiae cells were highpressure frozen, freeze-substituted and en bloc stained in lead and uranyl acetate prior to epoxy resin infiltration. To our knowledge this is the first demonstrated report of FIB-SEM resin infiltrated 3D cellular imaging using cryo-preservation without conventional pre-fixation/fixation. While en bloc lead staining (26) has been employed for serial block face imaging (30) our procedure demonstrated, as expected, excellent contrast via FIB-SEM. A comparison between TEM (Figure 1a) and BSE images from FIB-SEM (Figure 1b) showed the images to be very similar, but with somewhat reduced image detail and increased contrast of yeast cell walls with FIB-SEM. We then acquired serial FIB slices of yeast cells at $15 \mathrm{~nm}, 5 \mathrm{~nm}$, and 3 $\mathrm{nm}$ z-intervals, which were carefully chosen to assess the practicalities, limitations and impact these parameters had on image quality and structural integrity. While the $15 \mathrm{~nm}$ z-interval produced sufficient detail for many studies and the $3 \mathrm{~nm} \mathrm{z}$-interval provided the maximum spatial resolution of cellular components, $5 \mathrm{~nm}$ z-interval data was a reasonable balance of resolution, data size and the time required to collect data for an entire cell. The data set acquired at the $3 \mathrm{~nm}$ z-interval resulted in an XZ image (Figure 1c) that compared favorably to the XY FIB-SEM image (Figure 1b). A close inspection of the $\mathrm{XZ}$ image (Figure 1d) showed that relatively small structures, such as nuclear pores (arrows), were easily identified.

We report here, the ability to image through large cell volumes at reduced $\mathrm{z}$-intervals down to $3 \mathrm{~nm}$ isotropic voxels, which is a 6-fold reduction in z-interval from several previous reports using FIB-SEM in cells $(6,7,15)$ and comparable to a recent $5 \mathrm{~nm}$ isotropic FIB-SEM data set acquired from brain tissue (12). Our data extended upon this previous work by reducing the isotropic resolution to $3 \mathrm{~nm}$ and segmenting and quantifying numerous major cellular organelles from a single cell at $5 \mathrm{~nm}$ isotropic resolution using cryopreservation. Since it was not possible for us to directly measure the $3 \mathrm{~nm}$ milling as defined via software acquisition settings, we 
used three primary methods to validate the approach. First, as a rough visual inspection, the data showed no conspicuous distortions in aspect ratio when viewing the $X Y$ versus $\mathrm{Z}$ axis (Supplementary Video 5). Second, in order to empirically confirm the z-interval of $\sim 3 \mathrm{~nm}$, we used spherical vesicles within the resin embedded cell as an internal and sample specific control with a previously described cylindrical diameters method for measuring mean section thickness of TEM (31) and 3D FIB data $(6,11)$. Our results demonstrated a mean slice thickness of $3.603 \mathrm{~nm},(\mathrm{SD}=0.606, \mathrm{~N}=30)$. Consistent with this result, using $3 \mathrm{~nm} \times 3 \mathrm{~nm} \times 3 \mathrm{~nm}$ isotropic voxels, a $61 \mathrm{~nm}$ vesicle spanned 18 slices (Figure 1e). In comparison, the $15 \mathrm{~nm}$ z-interval data set required 6 slices to image section through a $74 \mathrm{~nm}$ diameter vesicle (Figure 1f). Third, we assessed milling consistency via continuous playback of the stacks of images (Supplementary Video 6 ) and while not flawless, overall the data showed relatively smooth transition from section-to-section for the data set. Furthermore, the gradual change in vesicle diameter at each step (Figure 1e) suggested that the BSEs that reached the EsB detector were restricted to a few nanometers from the surface. When low energy electrons hit a specimen, the penetrated distance depends roughly linearly on the electron's energy. The same is true with BSEs $(8,29)$ exiting the specimen surface which have a wide distribution in angle and energy since they may have experienced multiple scattering events and originated from different depths in the specimen. Conventional BSE detectors mounted below the objective lens typically collect nearly all BSE exiting the specimen surface and as a consequence $\mathrm{x}-, \mathrm{y}-$, and $z$-resolution are reduced. To overcome these limitations, an in-column EsB detector was employed (Supplementary Figure 1) similar to a previous report (12). BSEs with large scattering angles or that originated deep within the sample were restricted from reaching the $\mathrm{Es} \mathrm{B}$ detector either geometrically or by applying a bias energy at a filtering grid before the detector (Supplementary Figure 2). In this study, for $3 \mathrm{~nm}$ and $5 \mathrm{~nm}$ z-interval data, the primary beam energy was set to $1500 \mathrm{eV}$ and the energy filtering grid bias was set to $1000 \mathrm{eV}$, thus only BSEs having lost less than $500 \mathrm{eV}$ were allowed to pass through the grid and reach the EsB detector.

Modeling electron-sample interactions of biological systems is highly complex due to local chemical heterogeneity, including variations in structure and composition and the lack of a priori knowledge of the detailed cellular composition of our samples. Though the BSE radius, depth of
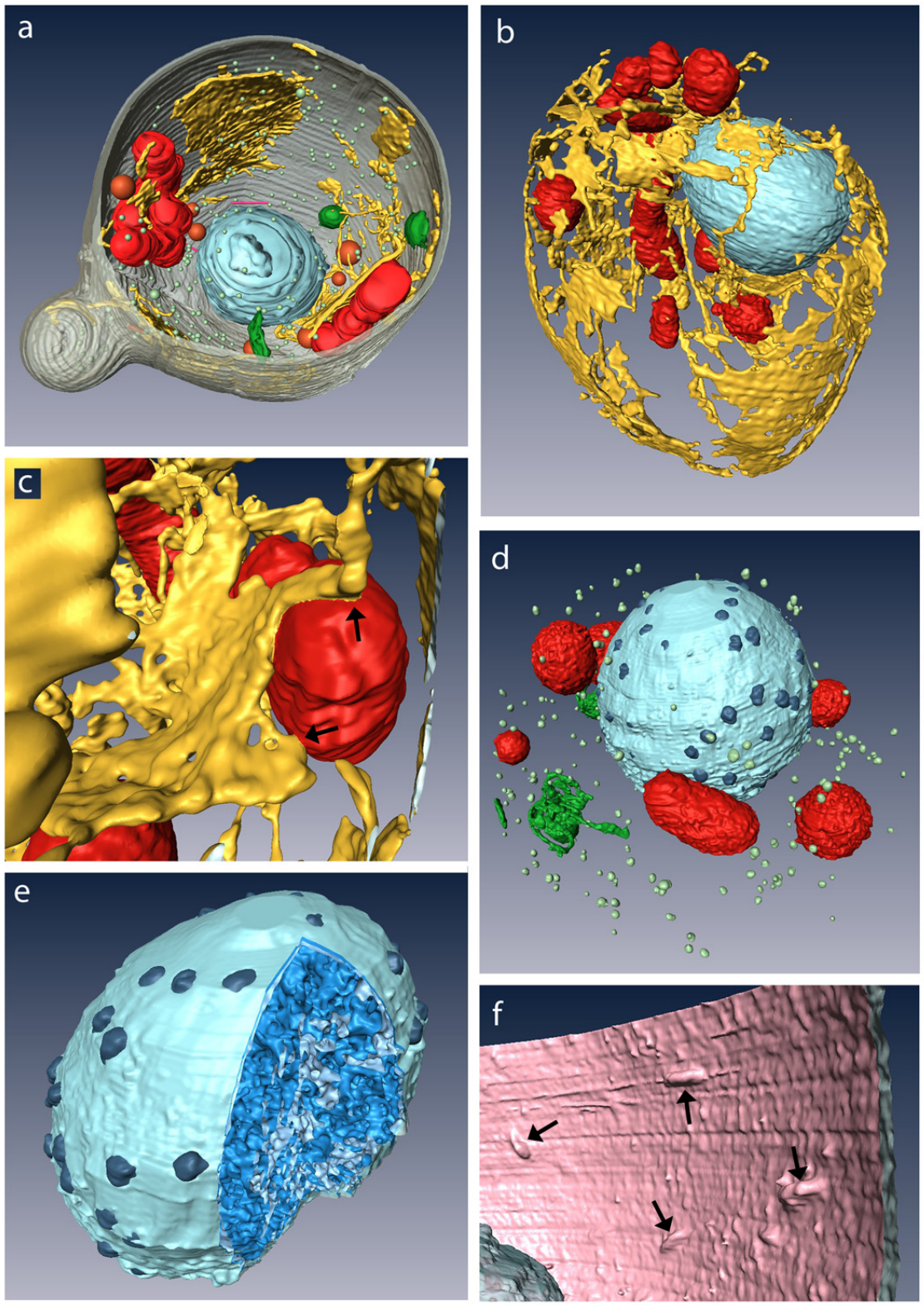

Figure 2. 3D segmentation. (A) $3 D 3.72 \mathrm{~nm} \times 3.72 \mathrm{~nm} \times 15 \mathrm{~nm}$ anisotropic FIB data set showed endoplasmic reticulum (ER; yellow), nucleus (light blue), cisternae (green), vesicles (light green), lipid droplets (orange), microtubules (pink), mitochondria (red) and cell wall (gray). (B) 3D segmentation of ER (yellow), mitochondria (red) and nucleus (blue) of a whole yeast cell at $5 \mathrm{~nm}$ isotropic voxels. Cell diameter $3.5 \mu \mathrm{m}$. (C) 3D segmentation of $5 \mathrm{~nm}$ isotropic data set with ER (yellow) and mitochondria (red) interconnectivity (arrows). (D) $3 D 3 \mathrm{~nm} \times 3 \mathrm{~nm} \times 3 \mathrm{~nm}$ isotropic FIB data set showed, nucleus (light blue), nuclear pores (dark blue), cisternae (green), vesicles (light green), and mitochondria (red). Cell diameter $3.2 \mu \mathrm{m}$ and final data set volume of $25.56 \mu \mathrm{m}^{3}$ (E) 3D segmentation of nucleus (light blue), nuclear pores (dark blue) and heterochromatin (bright blue) at $3 \mathrm{~nm}$ isotropic voxels. Nucleus diameter $1.2 \mu \mathrm{m}$. (F) 3D segmentation of plasma membrane (light pink) and plasma membrane invaginations (arrows).

emitted BSEs and BSE coefficients have been modeled previously in two pure metals (carbon and osmium) for $2 \mathrm{keV}$ beam with tilt (9), it is far from the real conditions of resin infiltrated biological cell systems. Here, we employed a simple and conservative composition, epoxy resin and osmium fixative $\left[\mathrm{C}_{21} \mathrm{H}_{25} \mathrm{ClO}_{5}\right.$ $+1 \%$ (atom) Os], to estimate the extent of electron penetrations within the epoxy embedded yeast. Monte Carlo simulation of electron-sample interactions showed that the BSEs for imaging were predominantly 3-4 $\mathrm{nm}$ below the surface $\left(\mathrm{E}_{0}=\right.$ $1500 \mathrm{eV}, \mathrm{E}_{\text {grid }}=1000 \mathrm{eV}$, Tilt $\left.=54^{\circ}\right)($ Figure $1 \mathrm{~g}$ ) as compared with over $20 \mathrm{~nm}$ in the case of conventional BSE detection for FIB without energy selection $\left(E_{0}=1500 \mathrm{eV}\right.$, 
Table 1. Quantitative Analysis of Cellular Components.

\begin{tabular}{|l|c|c|c|}
\hline Cellular component & Volume $\left(\boldsymbol{\mu} \mathrm{m}^{3}\right)$ & Volume Percentage & Surface Area $\left(\boldsymbol{\mu m} \mathbf{m}^{2}\right)$ \\
\hline Endoplasmic reticulum & 0.420643 & $2.2 \%$ & 31.403 \\
\hline Nuclear envelope & 0.227859 & $1.2 \%$ & 11.416 \\
\hline Heterochromatin & 0.577432 & $3.0 \%$ & 24.441 \\
\hline Euchromatin & 0.459362 & $2.4 \%$ & 20.716 \\
\hline Golgi & 0.022677 & $0.1 \%$ & 1.291 \\
\hline Mitochondria & 0.299339 & $1.6 \%$ & 6.949 \\
\hline Lipid droplets & 0.139214 & $0.7 \%$ & 2.692 \\
\hline Vesicles & 0.000256 & $0.0 \%$ & 0.025 \\
\hline Vacuoles & 1.480174 & $7.8 \%$ & 27.164 \\
\hline Cell Wall & 3.017192 & $15.9 \%$ & 67.115 \\
\hline
\end{tabular}

Quantitative analysis of volume, volume percentage and surface area of cellular components segmented in Avizo software.

Tilt $\left.=0^{\circ}\right)($ Figure $1 \mathrm{~h})$. This simulation confirmed the empirical results (Figure 1e) where the images of $3 \mathrm{~nm}$-interval slices consisted of mainly BSEs from those respective slices. The addition of atomic lead and uranium as part of simulations would only serve to further reduce the BSE signal collection volume (i.e., less than $3 \mathrm{~nm}$ ). Considering the complexity of biological samples and our inability to reliably determine the local heavy metal stain concentration, simulations with lead and uranium were not included, thus we emphasize our model estimates of electron penetration under the conditions employed were intended to be conservative.

As part of our characterization of the FIB-SEM high-resolution cell imaging approach, we sought to acquire, render and compare yeast volumetric data sets with 15 $\mathrm{nm}$ (Figure 2a), $5 \mathrm{~nm}$ (Figure 2b, c) and $3 \mathrm{~nm}$ (Figure 2d-f) z-intervals. FIB-SEM acquisition produced very large data sets and in the case of the $5 \mathrm{~nm}$ experiment, the resulting $3 \mathrm{D}$ volume was a $\sim 10 \times 7.5 \times 7.5$ $\mu \mathrm{m}^{3}$ and contained fine structural details of several yeast cells represented by about 5 billion voxels. This large amount of information was examined interactively with state-of-art graphics processing unit (GPU) accelerated volume rendering technology (Supplementary Figures 3, 4 and 5). Whole cells were extracted from the volume by creating a mask that isolated region of interests (Supplementary Figure 4). Detailed biological structures within the cell were segmented using a combination of automatic and interactive software algorithms prior to reconstruction. The 15 $\mathrm{nm}$ z-interval data set of a budding yeast cell with selected segmented organelles revealed excellent structural and positional detail; however, the z-axis stepping artifact of a spherical nucleus (light blue) and other structures was prominent due to the anisotropic resolution (Figure 2a, Supplementary Video 1). A $5 \mathrm{~nm}$ isotropic voxel data set of an entire yeast cell (Figure 2b, Supplementary Video 2) showed significant improvement in overall structural detail (i.e., mitochondria, endoplasmic reticulum, nucleus) with concomitant reduction in $\mathrm{z}$-axis stepping artifacts (i.e., nucleus) in this camera perspective. Furthermore, the spatial relationships of organelles with each other as well as sites of endoplasmic reticulum and mitochondrial interconnectivity (32) were readily documented (Figure 2c, Supplementary Video 3). To further push the systems capabilities while dramatically extending the total cell volume visualized, we acquired a data set at $3 \mathrm{~nm}$ isotropic voxel settings (Figure $2 \mathrm{~d}$ ) which permitted high-resolution $3 \mathrm{D}$ reconstructions of the nucleus, nuclear pore position and nucleoplasm/heterochromatin (Figures $2 \mathrm{~d}$ and $2 \mathrm{e}$ ), cisternae and vesicle distribution (Supplementary Video 4) and plasma membrane sights of invagination termed eisosomes (33) (Figure 2f). Images used in Figure 1c-f were derived from this same $3 \mathrm{~nm}$ volumetric data set and the raw $x-y$ aligned but unsegmented stack showed overall structural detail in the $x-y$ and $x-z$ axis with our approach (Supplementary Video 5). The accurate reconstruction of organelles in an entire cell allowed us to quantify targeted cellular structures, including but not limited to volume, volume percentage and surface area of various organelles (Table 1).

We conclude that this quantitative high-resolution FIB-SEM approach will open many new possibilities for visualizing the cellular organization of different cell and tissue types and complement other existing high-resolution approaches such as TEM tomography (19) and serial block face imaging (17). The segmentation of selected organelles and cellular components revealed their 3D spatial relationship with unprecedented z-resolution by FIB-SEM, approaching the gold standard of TEM tomography. Furthermore, the determination of organellar inter-relationships and connectivity $(32,34)$, improvements in nano-scale volumetric quantitative measurements/comparisons as well as the generation of targeted sub-cellular molecular distribution/localization maps using correlative microscopy (35) including photooxidation (36) are natural extensions of this methodology. Our overall goal to optimize conditions for highresolution $3 \mathrm{D}$ reconstructions of entire single cells allowed us to quantify and understand the spatial distribution of numerous sub-cellular structures (Figure 2 , Table 1). For the greatest accuracy of volumetric measurement with $3 \mathrm{D}$ FIB data sets, reduced milling intervals provide a distinct advantage (37) and for the yeast cell features, we found that this goal was best achieved by acquiring isotropic pixels at $\sim 5 \mathrm{~nm}$ or less. As with any approach which involves significant sample preparation and image processing (i.e., fixation, dehydration, resin embedment, image acquisition and analysis routines) consideration must be given to artifacts and 
limitations of each and interpretation adjusted accordingly. While $S$. cerevisiae served as a challenging biological specimen for optimizing sample preparation and FIB-SEM validation, we see no technical limitation for extending this strategy to larger cells at comparable pixel resolutions. However, significant improvements in FIB-SEM 3D resolution may likely require a technological breakthrough in SEM resolution itself, as well as improvements in sample stabilization and other environmental influences in the microscope and/or a method for tracking and correcting sample drift.

\section{Acknowledgments}

This project was supported by the Delaware INBRE program, with grants from the National Center for Research Resources - NCRR (5P20RR016472-12) and the National Institute of General Medical Sciences - NIGMS (8 P20 GM103446-12) from the National Institutes of Health.

\section{Competing interests}

Dongguang Wei is an employee of Carl Zeiss Microscopy, LLC a manufacturer of the Auriga 60 FIB-SEM used in this study. Shuang Zhang is an employee of Visualization Sciences Group, Inc. a developer of Avizo, the high-performance visualization software framework used in this study. All other authors declare that there is no conflict of interest.

\section{References}

1. Helmstaedter, M., K.L. Briggman, and W. Denk. 2008. 3D structural imaging of the brain with photons and electrons. Curr. Opin. Neurobiol. 18:633-641.

2. Micheva, K.D. and S.J. Smith. 2007. Array tomography: a new tool for imaging the molecular architecture and ultrastructure of neural circuits. Neuron 55:25-36.

3. Lehrer, J. 2009. Neuroscience: Making connections. Nature 457:524-527.

4. Schneider, G., P. Guttmann, S. Heim, S. Rehbein, F. Mueller, K. Nagashima, J.B. Heymann, W.G. Muller, and J.G. McNally. 2010. Three-dimensional cellular ultrastructure resolved by X-ray microscopy. Nat. Methods 7:985-987.

5. Bushby, A.J., K.M. P'Ng, R.D. Young, C. Pinali, C. Knupp, and A.J. Quantock. 2011. Imaging three-dimensional tissue architectures by focused ion beam scanning electron microscopy. Nat. Protocols 6:845-858.

6. Merchan-Perez, A., J.R. Rodriguez, L. AlonsoNanclares, A. Schertel, and J. Defelipe. 2009. Counting Synapses Using FIB/SEM Microscopy: A True Revolution for Ultrastructural Volume Reconstruction. Front Neuroanat 3:18.

7. Heymann, J.A., M. Hayles, I. Gestmann, L.A. Giannuzzi, B. Lich, and S. Subramaniam. 2006. Site-specific 3D imaging of cells and tissues with a dual beam microscope. J. Struct. Biol. 155:63-73.

8. Marko, M., C. Hsieh, R. Schalek, J. Frank, and C. Mannella. 2007. Focused-ion-beam thinning of frozen-hydrated biological specimens for cryo-electron microscopy. Nat. Methods 4:215-217.

9. De Winter, D.A., C.T. Schneijdenberg, M.N. Lebbink, B. Lich, A.J. Verkleij, M.R. Drury, and B.M. Humbel. 2009. Tomography of insulating biological and geological materials using focused ion beam (FIB) sectioning and low-kV BSE imaging. J. Microsc. 233:372-383.

10. Tanaka, K. and A. Mitsushima. 1984. A preparation method for observing intracellular structures by scanning electron microscopy. J. Microsc. 133:213-222.

11. Knott, G., H. Marchman, D. Wall, and B. Lich. 2008. Serial section scanning electron microscopy of adult brain tissue using focused ion beam milling. J. Neurosci. 28:2959-2964.

12. Knott, G., S. Rosset, and M. Cantoni. 2011. Focused ion beam milling and scanning electron microscopy of brain tissue. J Vis Exp:e2588.

13. Hekking, L.H., M.N. Lebbink, D.A. De Winter, C.T. Schneijdenberg, C.M. Brand, B.M. Humbel, A.J. Verkleij, and J.A. Post. 2009. Focused ion beam-scanning electron microscope: exploring large volumes of atherosclerotic tissue. J. Microsc. 235:336-347.

14. Armer, H.E., G. Mariggi, K.M. Png, C. Genoud, A.G. Monteith, A.J. Bushby, H. Gerhardt, and L.M. Collinson. 2009. Imaging transient blood vessel fusion events in zebrafish by correlative volume electron microscopy. PLoS One 4:e7716.

15. Murphy, G.E., K. Narayan, B.C. Lowekamp, L.M. Hartnell, J.A. Heymann, J. Fu, and S. Subramaniam. 2011. Correlative 3D imaging of whole mammalian cells with light and electron microscopy. J. Struct. Biol. 176:268-278.

16. Heymann, J.A., D. Shi, S. Kim, D. Bliss, J.L. Milne, and S. Subramaniam. 2009. 3D imaging of mammalian cells with ion-abrasion scanning electron microscopy. J. Struct. Biol. 166:1-7.

17. Denk, W. and H. Horstmann. 2004. Serial block-face scanning electron microscopy to reconstruct three-dimensional tissue nanostructure. PLoS Biol. 2:e329.

18. Hoenger, A. and J.R. McIntosh. 2009. Probing the macromolecular organization of cells by electron tomography. Curr. Opin. Cell Biol. 21:89-96.

19. West, M., N. Zurek, A. Hoenger, and G.K. Voeltz. 2011. A 3D analysis of yeast ER structure reveals how ER domains are organized by membrane curvature. J. Cell Biol. 193:333-346.

20. Jones, E.W. 1991. Tackling the protease problem in Saccharomyces cerevisiae. Methods Enzymol. 194:428-453.

21. Brachmann, C.B., A. Davies, G.J. Cost, E. Caputo, J. Li, P. Hieter, and J.D. Boeke. 1998. Designer deletion strains derived from Saccharomyces cerevisiae S288C: a useful set of strains and plasmids for PCR-mediated gene disruption and other applications. Yeast 14:115-132.

22. Sherman, F. 2002. Getting started with yeast. Methods Enzymol. 350:3-41.

23. Bernales, S., K.L. McDonald, and P. Walter. 2006. Autophagy counterbalances endoplasmic reticulum expansion during the unfolded protein response. PLoS Biol. 4:e423.

24. Liu, Y., M. Schiff, K. Czymmek, Z. Talloczy, B. Levine, and S.P. Dinesh-Kumar. 2005. Autophagy regulates programmed cell death during the plant innate immune response. Cell 121:567-577.

25. Walther, P. and A. Ziegler. 2002. Freeze substitution of high-pressure frozen samples: the visibility of biological membranes is improved when the substitution medium contains water. J. Microsc. 208:3-10.

26. Elliott, D. 2007. Serial Sectioning via Microtomy (or How to Get Over 100 Consecutive Serial Sections on One TEM Grid). Micros. Today 15:30.

27. Zhang, S., F.D. Maestra, N. Combaret, R. Klimentidis, P. Barthelemy, R. Albou, D. Lichau, and D. Bernard. 2011. The Analysis and Simulation of Rock Properties Using FIB-SEM and Vitrual Material Studio, NAFEMS World Congress, Boston, USA.

28. Joy, D.C. and S. Luo. 1989. An Empirical Stopping Power Relationship for Low-Energy Electrons. Scanning 11:176-180.

29. Pianetta, P. 2009. Section 3.2 Low Energy Electron Range in Matter, p. 5-10. In A.C. Thompson (Ed.), X-Ray Data Booklet. Lawrence Berkeley National Laboratory, Berkeley, California.

30. Tapia, J.C., N. Kasthuri, K.J. Hayworth, R. Schalek, J.W. Lichtman, S.J. Smith, and J. Buchanan. 2012. High-contrast en bloc staining of neuronal tissue for field emission scanning electron microscopy. Nat. Protocols 7:193-206.

31. Fiala, J.C. and K.M. Harris. 2001. Cylindrical diameters method for calibrating section thickness in serial electron microscopy. J. Microsc. 202:468-472.

32. Kornmann, B., E. Currie, S.R. Collins, M. Schuldiner, J. Nunnari, J.S. Weissman, and P. Walter. 2009. An ER-mitochondria tethering complex revealed by a synthetic biology screen. Science 325:477-481.

33. Walther, T.C., J.H. Brickner, P.S. Aguilar, S. Bernales, C. Pantoja, and P. Walter. 2006. Eisosomes mark static sites of endocytosis. Nature 439:998-1003.

34. Kornmann, B. and P. Walter. 2010. ERMESmediated ER-mitochondria contacts: molecular hubs for the regulation of mitochondrial biology. J. Cell Sci. 123:1389-1393.

35. Caplan, J., M. Niethammer, R.M. Taylor 2nd, and K.J. Czymmek. 2011. The power of correlative microscopy: multi-modal, multi-scale, multi-dimensional. Curr. Opin. Struct. Biol. 21:686-693.

36. Shu, X., V. Lev-Ram, T.J. Deerinck, Y. Qi, E.B. Ramko, M.W. Davidson, Y. Jin, M.H. Ellisman, and R.Y. Tsien. 2011. A genetically encoded tag for correlated light and electron microscopy of intact cells, tissues, and organisms. PLoS Biol. 9:e1001041.

37. Ritter, M. and P.A. Midgley. 2010. A practical approach to test the scope of FIB-SEM 3D reconstruction. J. Phys.: Conf. Ser. 241:e012081.

Received 5 March 2012; accepted 29 May 2012.

Address correspondence to Kirk J. Czymmek, Department of Biological Sciences, University of Delaware, 330 Wolf Hall, Newark, DE, USA. Email:kirk@udel.edu

To purchase reprints of this article, contact: biotechniques@fosterprinting.com 